\title{
Radio plasma fringes as guide stars: Tracking the global tilt
}

\author{
E. N. Ribak ${ }^{1}$, R. Ragazzoni ${ }^{2,3}$, and V. A. Parfenov ${ }^{4}$ \\ ${ }^{1}$ Department of Physics, Technion, Haifa 32000, Israel \\ 2 Astrophysical Observatory of Arcetri, Largo Enrico Fermi 5, 50125 Florence, Italy \\ e-mail: ragazzoni@arcetri.astro.it \\ 3 Max Planck Institute für Astronomie, Konigstuhl 17, 69117 Heidelberg, Germany \\ ${ }^{4}$ SI Vavilov State Optical Institute, 12 Birzhevaya liniya, St Petersburg, 199034, Russia \\ e-mail: vadim@optilas.spb.ru
}

Received 11 June 2001 / Accepted 7 July 2003

\begin{abstract}
We present a novel technique to alleviate the problem of the global tilt in artificial guide stars for adaptive optics. This technique is based on the registration of trails of radio-excited plasma spots caused by the atmospheric tilt. Following the time trace of the trails one can find and measure the tilt produced by atmospheric turbulent layers. Different methods were applied to estimate the extent of the trails. We describe results of computer simulations, showing the performance of the proposed approach.
\end{abstract}

Key words. instrumentation: adaptive optics - plasmas - techniques: interferometric - methods: data analysis

\section{Introduction}

In the past several years a number of $8-10 \mathrm{~m}$ ground-based telescopes have been built and more are now under construction. All of them are still limited by the distorting effects of the Earth's atmosphere, which limits their effective coherent aperture, and are thus provided with adaptive optics. Adaptive optics allows one to measure and undo the effects of turbulence in real time, delivering near-diffraction-limited performance at the infra-red and visible wavelengths. Many have detailed plans for adding artificial guide stars, which serve as reference beacons for precise measurement of the turbulence even when nearby stars are too weak for this purpose (Sandler 1999).

Unfortunately, artificial guide stars suffer from the problem of determination of the ascending beam global tilt, which is difficult to measure and remove from the descending beam. Because of reciprocity of propagation paths, a conventional synthetic beacon is unable to sense a full aperture tilt and can only be used to measure the higher-order distortions. For this reason, the problem of indeterminable tilt of artificial guide stars is recognised as a fundamental one to be overcome before applying a full adaptive optics correction for any type of seeing condition (Pilkington 1987). The current solution is to use a further natural star just for measurement of the tilt, complicating the optics and processing of the adaptive optics.

Since laser guide stars (LGSs) play a key role in the achievement of full sky diffraction capabilities at visible wavelengths, the efforts of the world astronomical community in

Send offprint requests to: E. Ribak,

e-mail: eribak@physics.technion.ac.il the past decade have (and still are) concentrated on the development of LGS technology. However, this solution has many problems, both practical and conceptual, slowing its application. For this reason, one of the authors has proposed an alternative approach to the creation of artificial reference source. This method is based on the use of visible plasma spots excited by fringes between intense radio waves (Ribak 1997, 1998). A few radio dishes or phased arrays are combined to have $1 \mathrm{~m}$ fringes, tens of kilometers above the telescope, the interference pattern spanning up to tens of meters. The air breaks down where the radio intensity is the highest, and creates plasma, visible in oxygen and nitrogen lines. Despite the attraction of the mentioned approach, the problem of lack of a useful tip-tilt signal is not solved in the case of radio guide stars (RGSs), just as it is not solved in the case of the LGSs. Therefore, the solution of the global tilt problem is very important for RGSs too.

The global tip-tilt problem has been attacked in several ways and now one can count more than ten techniques for tilt determination (Esposito 1998). A few of these techniques were tested experimentally on the sky. Namely, a statistical technique (Belen'kii 1995) was tested on Polaris using a Rayleigh laser beacon (Belen'kii et al. 1999). Shortly afterwards, a perspective based technique (Ragazzoni 1997) was tested by comparison on a few stars using a sodium laser guide star (Esposito et al. 2000). Both experiments are open loop ones, i.e. no attempt has been made for real-time correction of tip-tilt, but data have been collected and later reduced in order to establish the merits of the technique. Although both experiments show a remarkably good agreement with the expected results, 
it is worth pointing out that the correlation between the true and calculated tilt only amounts to $70-80 \%$. In addition the polychromatic LGS (Foy et al. 1995) is being actively pursued to achieve tip-tilt correction in a real adaptive optics system. The technique is based on non-linear excitation of the sodium layer and producing return light of two very different wavelengths. From the propagation dispersion of the two coloured beacons one can infer the absolute tip-tilt. Such polychromatic LGS has been observed experimentally on the sodium layer (Foy et al. 2000).

Recently several authors pointed out that wide-field-ofview adaptive optics (also known as multi-conjugate adaptive optics, or MCAO) (Beckers 1989) can be afforded by using natural guide stars (NGSs) alone (Ragazzoni et al. 2000a,b). It is clear that in such a framework the tip-tilt problem is virtually solved as there is no LGS at all. In the case of MCAO with LGS it is true that one can still apply some form of tomography (Ragazzoni \& Rigaut 1998) but this still requires some NGSs. All these approaches lead to a sky coverage that, although it can be remarkable under some conditions (Ragazzoni et al. 2002), still does not amount to the whole sky, especially for the shorter wavelengths. Tip-tilt solvers for systems not based on any NGS are still of great interest, especially in the framework of extremely large telescopes (Gilmozzi et al. 1998; Mountain 1997).

Thus more work is needed to provide a reliable system able to produce high Strehl ratios. The Kolmogorov turbulence is peaked at the very low frequencies, making the tip-tilt component very important. Hence only its very accurate removal can prevent significant deterioration of the achievable Strehl ratio (Rigaut \& Gendron 1992). However, there are theoretical suggestions that in the case of favourably smaller outer scales the tip-tilt contribution can be so low that, under special but not impossible circumstances, it will need no correction.

Even RGSs cannot solve the problem in full, since the pointing accuracy of the central fringe produced by the radio beams is limited due to their large wavelength. In addition, atmospheric effects are estimated to cause the interfering beams to wander at about $0.1 \mathrm{~m}$ at the elevation of $50 \mathrm{~km}$. On top of that, local winds up to $40 \mathrm{~m} \mathrm{~s}^{-1}$ might carry the plasma spot away, although its elevation can be chosen to be minimally affected by the wind. To make things easier, since the pulse repetition rate of the radio beams is chosen to leave residual plasma for the next pulse (to save on power), this repetition rate can be tuned to have a known spot drift. See the book by Gurevich et al. (1997, hereafter GBM97), for many details on the abovementioned considerations.

We suggest here a new concept for absolute tilt retrieval based on RGS technology. Because of the large scale of the radio fringes, this solution has direct implications also for the fields of radio astronomy, radio interferometry and optical interferometry, laser communications and power beaming (see below). In all these fields, there is a need for a local reference frame to help phase the elements of the radio dish, or co-phase the different dishes to serve as one coherent unit. The added capability to track tilts as proposed here will enhance these devices.

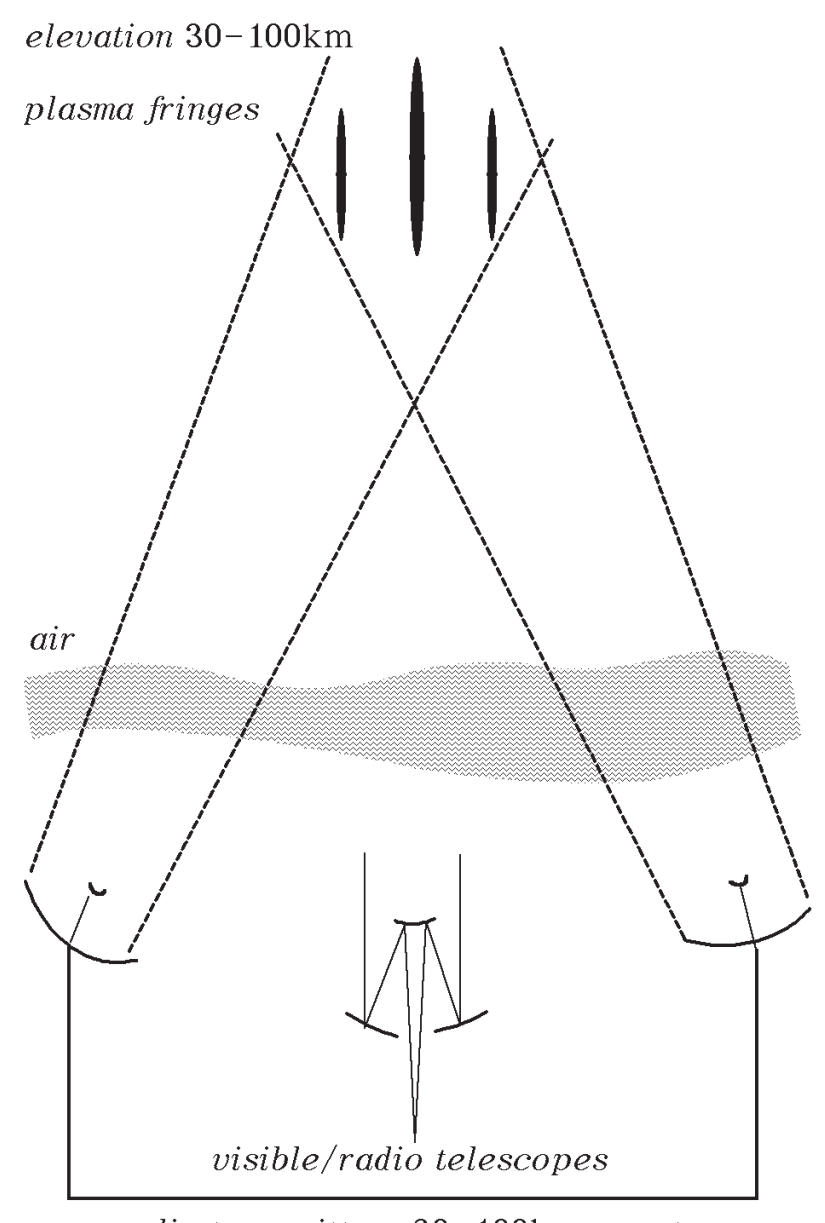

radio transmitters $30-100 \mathrm{~km}$ apart

Fig. 1. Radio guide stars are created as radio beams interfere and the air breaks down at the crests of the fringes.

\section{Radio guide stars}

The need for artificial guide stars has led to the suggestion to use inexpensive sodium lamps, and to image them on the sodium layer (Wirth \& Jankevics 1992). Since this image is too large to center on, interfering radio waves could quench the radiation along narrow fringes. This idea led to proposing that the interference itself could produce light by creating a very high local electric field in the crests of the fringes (Fig. 1). Such a strong field causes a breakdown of the thin air at high elevations, and the radiative recombination will be the artificial beacon (Ribak 1997, 1998). This is a kitchen table-top demonstration - every gas lighter produces a strong electric field, with a blue spark that flows through air as a result. These photons are the result of radiative relaxation of the excited plasma. The electric field required for breakdown is much lower at lower air pressures, but the lines are the same - nitrogen and oxygen lines. It turns out that this idea was tested before for other purposes. We detail now previous experiments for the interested reader. The description of our method continues in the next section.

Baily (1937), Gurevich (1972), Utlaut (1975) and others proposed to create an artificial ionosphere for reflection of radio waves above and behind the horizon. In the simplest scheme, a radio beam heats up and ionizes or creates a 
break-down in the atmosphere. At an elevation of 30-60 km a patch of plasma is thus created. Efficient reflection of the distant radio waves occurs only at a very high plasma density, so the idea was improved to have two radio beams interfering in order to create fringes. The electric field density culminates in the fringes where ionization starts, and the few electrons multiply very fast. The ionized fringes serve as a radio Bragg reflector, a more efficient scheme than mere return from a crude plasma volume (Gurevich 1980). In a laboratory experiment, clear visible fringes were apparent and served to trace the plasma distribution (Kuo \& Zhang 1990). Among other applications, the use of artificial ionization was suggested as a means to create a high power nitrogen laser $\left(100 \mathrm{~kW}\right.$ inside $\left.1 \mathrm{~m}^{2}\right)$ in the atmosphere, shining down on the ground (GBM97). Fringes are created between moving microwave beams, with a crossing area moving down at the speed of light. The advancing breakdown regime is a source of spontaneous followed by stimulated emission. In laboratory tests $3 \mathrm{~kW}$ radiation at $337.1 \mathrm{~nm}$ were produced (Vikharev et al. 1991). Full description of the physical processes involved can be found in GBM97, and physical and engineering aspects are described in Gaponov-Grekhov \& Granatstein (1994). The detailed set of parameters of the system for production of visible fringes (power of transmitter, diameter of dishes or phased arrays, etc.) for astronomical purposes depends upon a number of variables (geographical constraints, available infrastructure, etc.) which have to be taken into account in a full engineering design as discussed in length in these same references, to which the interested reader is directed.

One should be concerned by other lines, and especially by the background produced by the plasma, as such light can hamper detection of faint objects if not properly filtered out. Luckily previous experiments did look for lines others than the ones mentioned here, and found them insignificant. In principle, however, these could be removed by a proper set of blocking filters, unless they are numerous. As this would be the case of $\mathrm{OH}$ lines we point out that given the altitude where RGSs are produced, their existence is very unlikely.

The ionosphere around $250 \mathrm{~km}$ (Bernhardt et al. 1988) was studied by similar methods: radio heating of a large volume, by a single radio dish, creates plasma and cavitation of the heated volume. The result is enhanced air glow, as well as a typical radio signature, over tens of kilometers. Electrons ionized and/or accelerated in the heated volume spiral out of it, along the earth's magnetic field, and create an illuminated regime when they hit denser neutral species. However, at these elevations (above $150 \mathrm{~km}$ ) the diffusion of the plasma and the fast winds disperse and shift swiftly any created patterns. In these experiments, relatively low power was required (10 kW-2 MW), in a continuous mode. At lower elevations, the wavelength has to be shorter (microwave), and because its absorption decreases as more plasma is created, it is necessary to utilize pulses rather than continuous radiation. In a recent experiment, a structured enhancement of the $557.7 \mathrm{~nm}$ line was observed at $90 \mathrm{~km}$, even though the radio beam was heating a higher volume (Kagan et al. 2000).

The ionospheric air glow is very patchy. This is due to the density variations of the excited volume. In addition, filamentation was observed in the heated area, as well as in laser sodium guide stars. It is not clear what is the typical spatial frequency of the patches and what is their contrast. They have only been resolved down to the ten meter scale, somewhat consistent with theoretical predictions. The visible natural intensity (night glow) is usually few tens of Rayleighs, and in the experiment values above 250 Rayleighs $\left(\approx 16^{\mathrm{m}} / \operatorname{arcsec}^{2}\right)$ were also produced.

The choice of beacon elevations is important from the point of view of adaptive optics: the higher the better, provided the pattern is stable enough and of high enough contrast on the few arcsecond scale. Above $200 \mathrm{~km}$ the random pattern can still be used as a reference source, much in the same way that the solar surface serves for adaptive optics. Below $100 \mathrm{~km}$ the density of the atmosphere is high enough that the plasma mean free path $(\mathrm{a}$ few $\mathrm{cm})$ is much shorter than the required fringe period, which is less than $2 \mathrm{~m}$ (Baharav et al. 1994, 1996). Thus the luminescent fringes follow the radio-heated ones. Laboratory experiments show that the most power-efficient air break-down occurs around 0.5-5 Torr (Kuo \& Zhang 1990), corresponding to an elevation of $30-80 \mathrm{~km}$. Although this paper focus essentially on the use of RGSs as a way to produce a suitable reference beacon at a general altitude, the sodium layer at 87$95 \mathrm{~km}$ is also a respectable compromise (now excited by radio beams and not by lasers). In addition, the created plasma field can break up sodium dust particles into atoms and increase the returned signal (Wuerker 1997).

A portion of the sodium layer was irradiated by a $1.35 \mathrm{MHz}$ transmitter with a resulting enhancement of the sodium lines by 100 Rayleighs (Lyakhov et al. 1984), corresponding to $m_{v}=$ $17 / \operatorname{arcsec}^{2}$. No details were given for the actual power used or the spot size created, except that it was measured from a distance of $90 \mathrm{~km}$, at an elevation of 80-100 km. Natural and laser guide stars need to be brighter than $m_{v}=12-14$. To achieve this, single lasers need be brighter than 8-12 W. Laser fringe systems, which measure more than one atmospheric layer, require 200-500 W (Baharav et al. 1994, 1996). However, these estimates can be reduced drastically by employing an optical fringe demodulation scheme (Ribak \& Ragazzoni 2001) to $m_{v}=11-13(11-16 \mathrm{~W})$. This is close to the theoretical limit, set by the number of degrees of freedom, or $r_{0}$ patches, to be measured. In two conjugate layers there are only $20-30 \%$ more $r_{0}$ patches than one integrated volume. Thus by reducing the plasma spot size by a factor of one hundred with respect to Lyakhov et al. (1984), the integrated surface brightness could reach the required level for a full sky coverage. This reduction in size can be achieved by employing phased arrays and interfering beams, and wavelengths shorter by orders of magnitude.

\section{The tilt tracking technique}

Tilt, produced by atmospheric turbulent layers, can result in a trail of the spot of the artificial guide star visible from the telescope. The same effect occurs due to differential piston between interfering radio beams. In considering this spot movement, we pose a question: is it a positive or a negative effect and can it be used to break down the reciprocity and to sense a full aperture tilt with an artificial guide star? 
To answer this question, let us suppose that the upward interfering beams exciting the beacon are characterised by an upward wandering $\boldsymbol{b}(t)$. In what follows, we only consider the central interference crest, which we term loosely as fringe. By using distant phased arrays we can control the shape of the interference pattern and create distant sharp maxima. Averaging of more maxima (which move in unison but differ in plasma detail) can only improve the method discussed below. Let us also suppose that the excited spot has a certain decay time $t_{0}$. In other words, if one excites the beacon with a single short pulse of intensity $I_{0}$, one will observe a beacon intensity that changes with time accordingly to

$I(t)=I_{0} \exp \left(-t / t_{0}\right)$.

For the mesospheric sodium layer, excited by laser light at the same $D_{1}$ and $D_{2}$ lines (Happer et al. 1994; Sandler 1999), $t_{0}$ is few tens of nanoseconds. The effect of the trails caused by turbulent atmosphere tilt is negligible and hence useless. However, in exciting the oxygen or nitrogen in a plasma cloud using radio waves, the decay time $t_{0}$ can take much longer values. Because the recombination of plasma varies according to the density of the atmosphere, the decay time will depend upon the height of the excitation. At the altitude of $60 \mathrm{~km}$ the decay time of plasma is of the order of $t_{0} \approx 0.01 \mathrm{~s}$, comparable with the time required for the excited beam to be detected above the noise level. The repetition rate is chosen so as to leave exactly this residual plasma for the next pulse. Hence, we will see simultaneously the recently excited spot and some residue of previous spots next to it. As a time-series, this looks like a bright spot with a trail behind it. This is similar in appearance to an over-exposed image intensifier showing trails behind bright moving spots. Therefore, in the case of RGS the trails of the excited plasma spot, produced by tilt, will be visible from the ground-based telescope.

The elevation of the plasma can be chosen by the transmitting phased arrays. As a result the local air density will vary, and with it the decay time $t_{0}$. Thus it can be chosen to be of the same order of the Greenwood frequency. However, there is no need to have $t_{0}$ locked to some specific frequency related to the atmospheric parameters.

Except for an ionospheric beacon (Ribak 1997, 1998), the spot and its trail are always contained within an isoplanatic patch. In this situation, we will see the evolution of the beacon. Because of the non-zero decay time $t_{0}$, we will see a sort of evanescent trace that follows the beacon movements. The direction and extension of portion of the trail closer to the beacon is an indication of the variation of the upward tilt. A slowly moving upward tilt will produce a short trail, a fast moving upward tilt will produce a longer trail. For instance, looking at the brightest point in the trail (the current beacon), of brightness $I$, and at an adjacent point of brightness $I / e$, one will see exactly an angular separation of

$\boldsymbol{s}(t)=\boldsymbol{b}(t)-\boldsymbol{b}\left(t-t_{0}\right)$.

This is, roughly speaking, the derivative of $\boldsymbol{b}(t)$. More precisely, this is a finite difference of $\boldsymbol{b}(t)$ with respect to $t_{0}$ (Fig. 2). In any case, by integration or numerical summation one should be

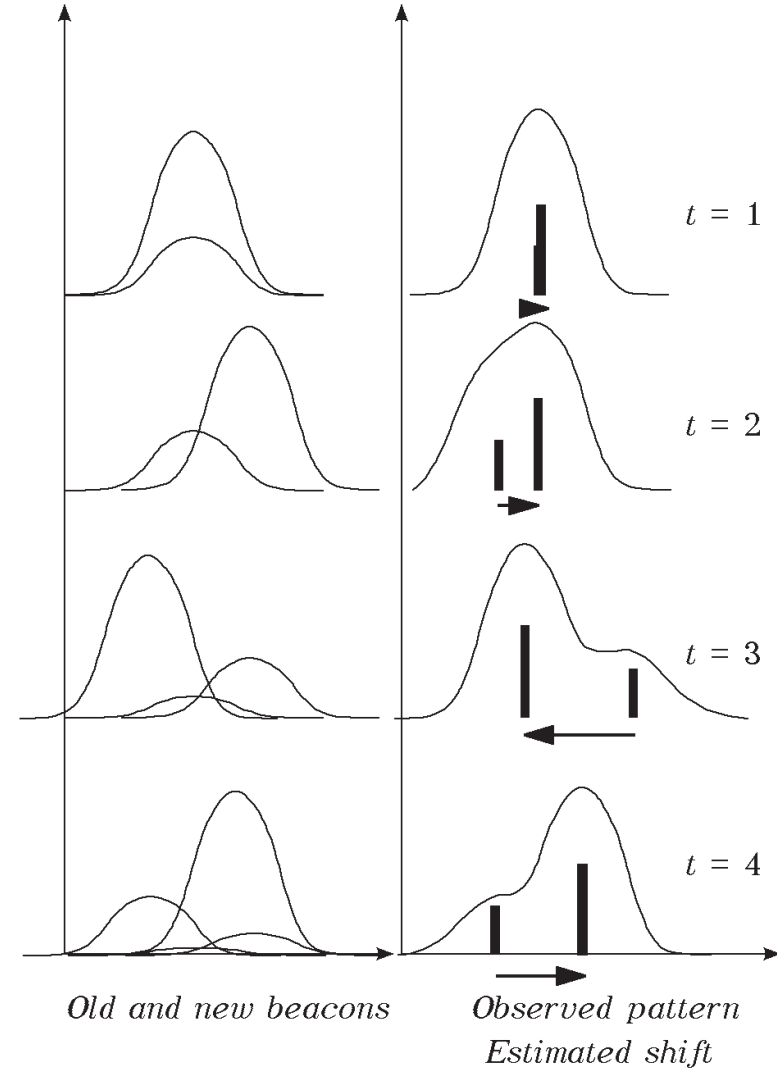

Fig. 2. Decay and motion of the plasma spot. On the left are shown the recent few spots, and on the right their (noiseless) appearance. The two bars and arrows are estimates from this observed pattern, where the global tilt is not known.

able to retrieve $\boldsymbol{b}(t)$. Each measurement of the finite difference will be affected by some error and the integration error will grow with time leading, at a certain time, to some unacceptable value. If this happens after long time (compared to $t_{0}$ ), one can lock onto a much fainter natural guide star and close the low frequency loop there. Such an approach was discussed in detail previously (Ragazzoni 1996).

Using the assumption that $\boldsymbol{b}(t)$ is always much smaller than the isoplanatic patch, the measurement of the trail extent and position angle is only marginally affected by the downward tilt, because both $\boldsymbol{b}(t)$ and $\boldsymbol{b}\left(t-t_{0}\right)$ are observed at the same time and are in the same isoplanatic patch. Hence, this downward tilt has no effect on the differential evaluation of the derivative of the upward tilt.

Also, vibrations of the telescope structure are directly observed (Foy et al. 1995), as the upward beacon is not produced by the observing telescope at all, but by interference between distant radio dishes, whose detailed pointing is almost meaningless in the position of fringes.

Therefore, the full aperture tilt of the atmosphere can be found and measured following the time trace of the trails of the radio beacon. It means that the proposed technique permits the sensing of the full-aperture tilt of the atmospheric wave front distortions using a RGS, eliminating one of the fundamental limitations of adaptive optics. 


\section{Signal and noise estimates}

We wish to find out the required intensities for our scheme to be applicable. The (angular) size of each fringe is $\lambda_{\mathrm{r}} / b$, where $\lambda_{\mathrm{r}}$ is the radio wavelength and $b$ the base line. The centroiding error is $\lambda_{\mathrm{r}} / b \sqrt{N}$, where $N$ is the number of photons and photon shot noise is the only relevant noise source. The number of measurements is the ratio of the total measurement time $\tau$ to the single frame integration time $t_{0}$ (which is the smaller of the Greenwood rate and the plasma decay rate). As the number of measurements grows as $\tau / t_{0}$, this centroiding error grows as $\sqrt{\tau / t_{0}}$. The number of photons in a single integration, $N$, is related to the number of photons per second, $N_{0}$, through $N=N_{0} t_{0}$. The total centroiding error is

$\sigma=\frac{\sqrt{\tau} \lambda_{r}}{t_{0} \sqrt{N_{0}} b}$

This error has to be smaller than $\lambda / \alpha D$, where $\alpha \approx 2.3$ (Sivaramakrishnan et al. 1995). Solving for the number of photons per second, $N_{0}$, we get

$N_{0}>\frac{\alpha^{2} \tau}{t_{0}^{2}}\left(\frac{D \lambda_{r}}{b \lambda}\right)^{2}$ photons/s.

To get the required brightness in Rayleighs we divide this number by $10^{10}$ photons for SI units, by the aperture area and by the fringe angular area:

$B=\frac{N_{0}}{10^{10} \pi(D / 2)^{2}\left(\lambda_{r} / b\right)^{2}}>\frac{6.7 \times 10^{-11} \tau}{t_{0}^{2} \lambda^{2}}$.

For the sodium line we take as representative values an integration time $t_{0}=0.01 \mathrm{~s}$ and total integration time of $\tau=0.1 \mathrm{~s}$, obtaining $2 \times 10^{5}$ Rayleighs from a single fringe crest, much in line with the estimates in Sect. 2. This integration time should allow for full sky coverage provided that a faint NGS in the isoplanatic patch is used to corroborate the measurement (Ragazzoni 1996). In fact as $t_{0}$ is a short time with respect to the inverse of the Greenwood frequency (Glindenmann 1997; Parenti \& Sasiela 1994; Tyler 1994), $\tau$ is not as small and the continuous centroiding of the faint star is used to recover from the cumulative errors in the tilt derivative estimates on $s$. Inclusion of all the crests will reduce this requirement even more. This is because the number of fringes in each dimension is proportional to the ratio of the base line $b$ to the single aperture diameter $d$. For $(b / d)^{2}$ fringe crests the error will drop by $b / d$ and the total plasma pattern area will grow by $(b / d)^{2}$, with an attendant drop in the necessary flux by a factor of $(b / d)^{3}$.

\section{Simulations}

To check the proposed approach we ran many computer simulations. Following the previous section, we assume that we have enough photons to identify precisely the centroid of the data. Now we wish to see if we can reduce the demands in order to follow and integrate on the spot trails to reduce the tilt problem. Specifically, we generated a series of RGS spots using a plain Kolmogorov spectrum for the upward tilt, common to the three radio beams involved. The drift in the tilt (and hence the trail) of the radio beams can be as small as $0.1 \mathrm{~m}$ (GBM97). Even a smaller drift can be detected by extending it intentionally. Since the radio beams are interfered to create bright fringes, all one has to do is to change the phase of the beams relative to each other in an oscillatory manner. Notice, however, that the shift of the spot cannot be larger than its diameter, since each radio pulse re-vitalises the plasma from the previous pulse, this being more power efficient than a continuous excitation (GBM97). The shape of the plasma spots depends on the chosen wavelength, elevation, and distance between transmitters (GBM97), and can range between a few decimeters and a few decameters (Ribak 1997, 1998). We used a Gaussian spot profile, of standard deviation varying between 75 and $200 \mathrm{~cm}$.

In the simulation, we created a single plasma spot; for three transmitters one would get at least three, around the telescope, but we only wish to prove the method here. The next pulse will create a new nearby spot, while the older one is reduced by a decay factor $d$, chosen between 0.1 and 0.7 (i.e. with decay times different from $t_{0}$ ). These values were selected following exploratory simulations to define the relevant range: a larger decay would give too small a separation $s$ while a very small decay will make the tail too extended to be centred accurately. The next shot will add yet another new spot, with the two older ones decayed again by the same factor. Some snapshots of these simulations are shown in Fig. 3. Between shots, we moved the beam using a Kolmogorov spectrum of tips and tilts, with a preset standard deviation varying between 1 and 3 pixels as measured tangentially with respect to the line of sight of the telescope. In the analysis of the fringes, we added some Poisson noise to the detected signal: we show results for 20 to 2000 photons at the brightest pixel. Other noise sources, such as detector additive noise, or non-homogeneous plasma, were neglected. Specifically, at high pressure (low elevation), non-linear growth of the plasma patches might not follow the shape of the peak of the generating fringes (GBM97). Higher up, this shape quickly diffuses into a Gaussian.

All calculations were performed in terms of pixels only, where we arbitrarily define the scale of each pixel to be $\approx 0.1 \mathrm{~m}$ at the plasma elevation. Further studies need to be carried out to find the best pixel size in order to maximise the accuracy. We now have a sum of two Gaussians, one strong and one weak, and even weaker residues of older Gaussians (Figs. 2, 3). We have to identify the Gaussians, so as to detect the most recent one and the distance from it to the previous one. A foremost requirement is speed of calculation, in view of the limited time between pulses. This is a difficult task, even without including noise in the signals. We tried a number of ways to analyse the data.

(a) We first identified the brightest point in the pattern. This is the approximate location of the current spot. We subtract a Gaussian from the intensity pattern, and locate the previous spot as being the next brightest point again. This is similar to the CLEAN algorithm used to deconvolve radio maps (Thompson et al. 1986; Perley et al. 1985), except that we stop after only two iterations. The resolution is limited to integer pixels. 


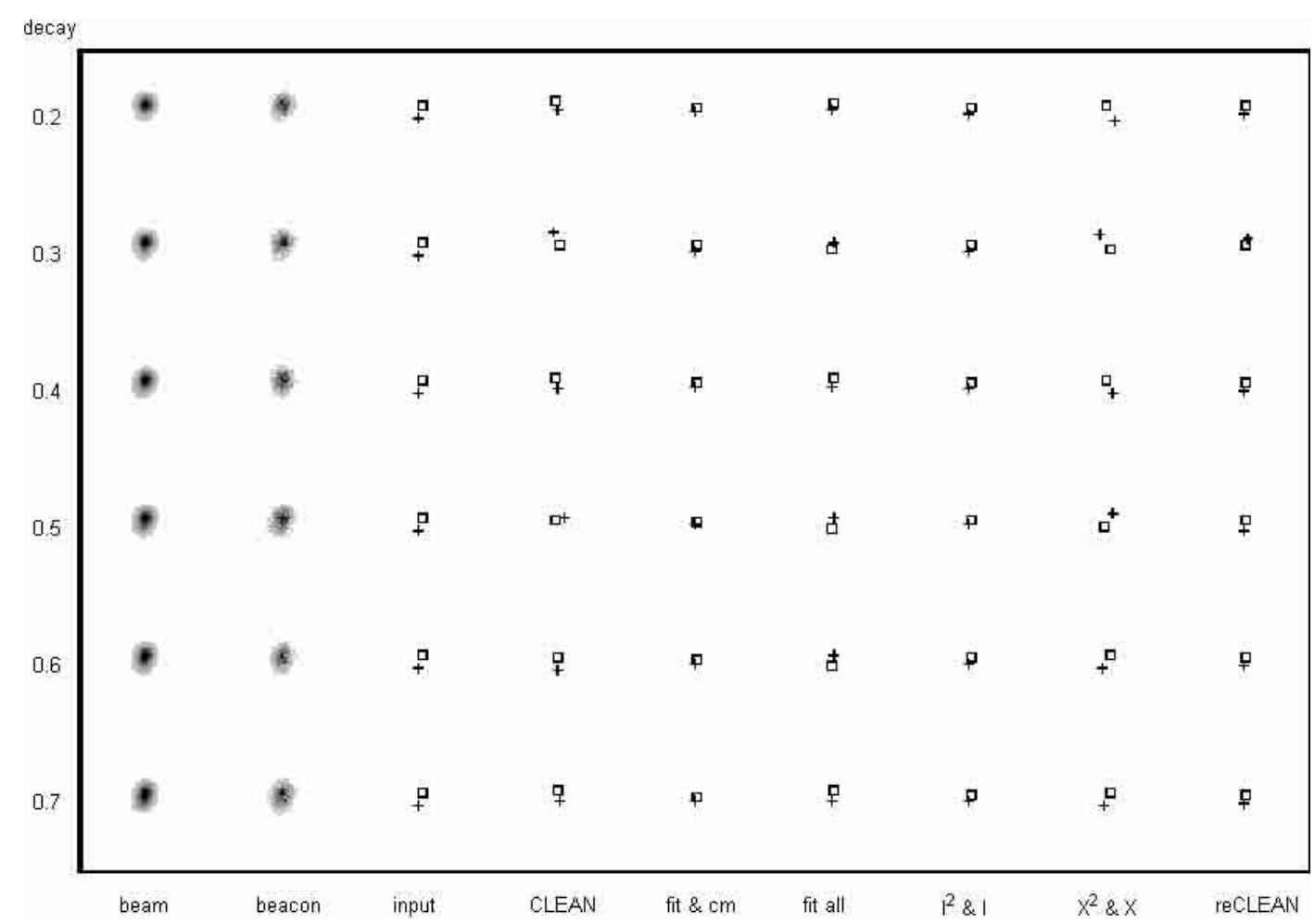

Fig. 3. Sample simulation step: the location of the previous (cross) and current (box) beacons are the same but the decays are different (top to bottom). From left: noiseless signal, signal with noise, true locations, and results of the six estimators. The mean number of photons at the brightest pixel was twenty.

(b) Next, we tried to use optimisation: we fitted one Gaussian near the brightest pixel, and subtracted it as before. The older spot was located by finding the center of gravity of the residual.

(c) The first two methods suffer from bias in the choice of the first spot, a bias introduced by the nearby second spot. To avoid this bias, we fitted two Gaussians simultaneously. The optimisation process involved in the fitting is rather slow compared to $t_{0}$.

(d) Another way to tell the new and old spots apart is by comparing the first moment of the intensity pattern and the first moment the square of the intensity pattern. Because the older spot is weaker, it will show less on the squared image. Let $d$ be the decay factor (ratio of the previous to current intensity), and the first and second intensity moments be $i_{1}$ and $i_{2}$. Call for short the estimates for the previous, current, and separation, $a, b$, and $s=b-a$, respectively. Now we get

$s=\left(i_{1}-i_{2}\right) /\left[d^{2} /\left(1+d^{2}\right)-d^{1 / 2} /\left(1+d^{1 / 2}\right)\right]$,

$b=i_{1}-s d^{2} /\left(1+d^{2}\right)$.

(e) In an alternative analysis, we found the first and second coordinate moments of the elongated spot. Assuming a Gaussian profile for each spot, we found the analytical values of these moments along the two Cartesian axes. Thus we could find the locations of the two recent spots and hence their separation. Including the location of the brightest spot in the pattern broke the central symmetry of these moments. Let $v$ be the variance of the Gaussian spot, $x_{1}$ and $x_{2}$ the first and second moments, and $x_{3}$ the location of the brightest spot. The old and new spots are at

$$
\begin{aligned}
& a=x_{1}-\operatorname{sign}\left(x_{3}-x_{1}\right)\left[\left(-x_{1}^{2}-v+x_{2}\right) / d\right]^{1 / 2}, \\
& b=x_{1}(1+d)-a d .
\end{aligned}
$$

(f) Since the spots tend to merge, it is possible to sharpen the images digitally. We used a Wiener deconvolution scheme, in which the theoretical shape of the spot was used as the object, and the noise has Poisson statistics (Christou et al. 1986; Ribak 1986). In the Fourier domain the deconvolved image is

$$
I_{\text {deconv }}=I_{\text {source }} S^{*} /\left(S S^{*}+N\right),
$$

where $I_{\mathrm{deconv}}, I_{\text {source }}$ and $S$ are the Fourier transforms of the deconvolved image, the original image, and the spot profile. $N$ is the mean power spectrum of the noise, and $*$ symbols conjugation. After deconvolution, the brightest and next brightest spots are identified, as in (a). The accuracy is limited again to integer pixels.

After identification of the two last spots in the pattern, we calculated their distance. We integrated this quantity for a large number of pulses. Although we have quite good knowledge of the statistics of the beam wander, we did not include them in the calculation as a prior. This allows one to include other sources of tilt, such as mechanical vibrations, as well as intentional scanning of the beams. In the simulation, we used the 


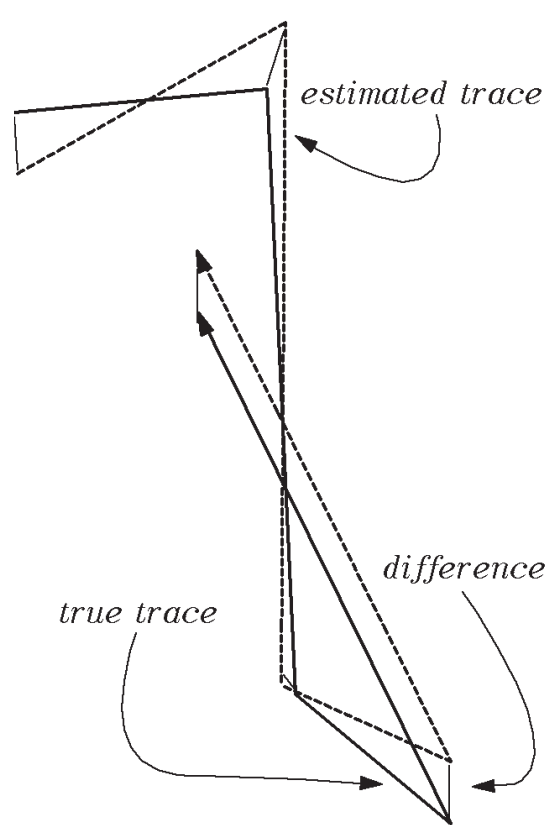

Fig. 4. Calculation of the error. At each step the difference between the estimated trace and the true trace is taken. This is the current error, equivalent to subtraction of estimated and true displacement from the previous step.

known Gaussian spot shape for fitting it in the different methods. In the experiment, this profile will have to be measured.

In Fig. 3 we see the true location of the trail as created by the radio beacon in the sky, and the calculated location from the data reduction using the different schemes. It should be stressed that although the image included the whole history of the drifting spot, the fitting was made only to the two recent spots; the rest of the trail, not accounted for the the calculation, reduced the quality of the fit. As shown next, the method works best when the trail extent is the longest and the spots are nearly separated, when the decay in time is the least, and when the noise is negligible. Identification of the brightest location is always quite accurate, but that of the previous one is lacking. This is the main source of error. We should remark that discretisation errors, in some of the methods, grow with the square root of the integration time. Still, we could see that the numerical integration did follow the actual beam with time even for quite unfavourable conditions.

We compared the six methods for different decay values. In this case, the Gaussian spot had a standard deviation of 3 pixels, and the jitter, random or intentional, a standard deviation of 4 pixels. The mean signal at the brightest pixel was twenty photons, and the simulation included 200 steps. If the true and estimated translation vectors (between old and new spot, Fig. 4) at step $i$ were $t_{i}$ and $e_{i}$, what is shown is $\left[\left|t_{i}-e_{i}\right|^{2} / \Sigma i\right]^{1 / 2}$. The results are shown in Fig. 5: the two methods with the least error (b and d) are also the methods that tend to slightly underestimate the translation vector - the other methods slightly overestimate it. At higher fluxes, the accuracy improved proportionately, with the more precise methods (b and d) keeping their edge over the other methods.

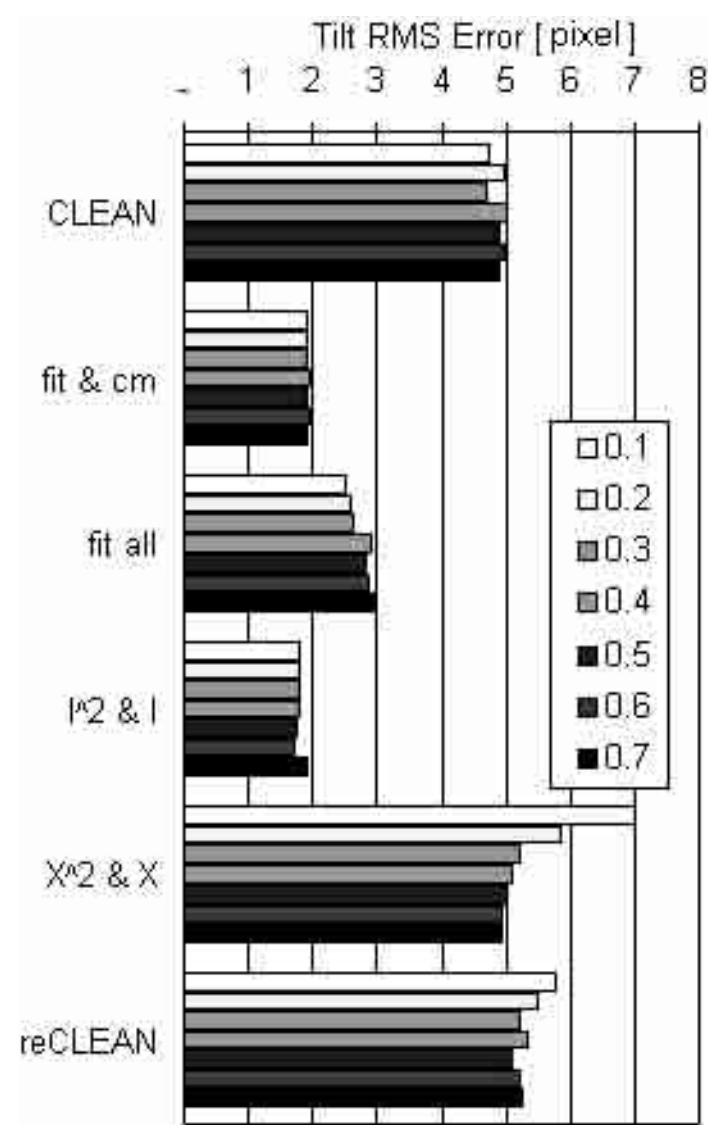

Fig. 5. Comparison of methods at different decay values ( 0.1 to 0.7 for each method), for signals up to twenty photons/pixel. The standard deviation of the beam jitter was 4 pixels, and that of the beacon size 3 pixels. Better models of the physical processes should improve all results.

\section{Parameters of the method}

The process of the creation of trails of RGSs may be more complicated than simulated here, since atmospheric wind dispersion and general diffusion can also result in this effect. These processes tend to spread even the single beacon (GBM97). In particular, the maximum speed of winds at altitude of about $60 \mathrm{~km}$ (where inter-pulse time is about $10 \mathrm{~ms}$ ) and latitudes between 30 degrees north and 30 degrees south (where most telescopes are located) can reach a reasonable value of about $20 \mathrm{~m} \mathrm{~s}^{-1}$. Tuning of the plasma to an altitude of slower wind is possible. However, in this study we have not introduced any influence of wind on the beacon layer for simplicity. We believe that wind, if constant in direction and intensity, will introduce an additional apparent constant value in the derivative estimate. This can be removed easily in the real-time reduction process, because it is a constant offset in the derivative, estimated at each update loop on the faint natural guide star.

How does the distance between the old and new plasma spot affect our results? Of course, the further the points are the easier they are to separate numerically. If this separation is too small, we can enhance it by scanning the relative phases of the interfering beams, and thus moving the fringes across the interference pattern. Unfortunately artificial sweeping of the spots, or by atmospheric propagation phase and local wind, 
cannot exceed a fraction of the spot size: beyond this distance, the seed of ionised air from the last pulse will not be enough to initiate the new breakdown. This is especially important at lower elevations, where recombination is fast and complete. In the simulation we replaced intentional sweeping by random tilts, created by pure atmospheric spectrum of intentionally large extent.

The effect of noise was also investigated. Since there are only rough estimates for the number of photons available (Sects. 2, 4 and GBM97), we included in the simulation from a few to an infinite number of photons. We found out that down to 20 photons (at the brightest pixel) we could still locate the current and previous beacon, but below that the signal deteriorated too fast. In addition, Poisson noise caused over- or underestimation of the locations and translation (difference) vectors, because this noise was not included in the simple model of the system and the resultant estimator methods (a)-(e).

The effect of decay of the plasma between pulses is also important. Since there are again only rough estimates for this decay, we assumed the intensity of the previous pulse is between 0.1 and 0.7 of the recent one. The stronger the decay, the easier it is to find the location of the two spots. This is partly because in all six methods we assume (in the calculation) only two such spots, and neglect the rest of the history, even though it appears in the image. Notice that in extreme decay the energy in the past spots can be comparable to the recent one(s), which adds to the error (private communication, anonymous reviewer). However, when the signal is too weak, a strong decay means low signal-to-noise ratio for the previous spot.

As a result of these simulations, we can say that for each elevation one should be able to find the optimal choice of parameters. At low elevations $(30-50 \mathrm{~km})$, the decay is rapid, so the pulses should be close. If the overlap is then too much, constant wind or artificial sweeping are required. Further up more power is needed, the plasma decays more slowly, and the pulses can be spaced apart, but the weaker signal might be a limitation. Above $100 \mathrm{~km}$ the plasma pattern is set by other parameters (GBM97) such as magnetic field lines and is much less controllable.

\section{Conclusions and applications}

We showed that the scheme of tracking the trails of plasma spots in order to reduce the tilt problem is indeed valid for the simple assumptions made here. We tested a number of methods to reduce the data and showed that it is possible to follow the tilting beam with time. The most accurate method was fitting of a Gaussian to the plasma spot, and calculation of the center of gravity of the residual. Better results are possible by extension of the fit to even older spots, and by fine-tuning of the chosen parameters for the fit.

The utility of the method depends strongly on the actual flux available from the plasma spots. Even for a very small number of photons we are able to trace the spot to within 1 arcsec, and for an essentially infinite flux, down to 0.03 arcsec. While there seem to be enough photons to locate the centroid accurately enough, the cumulative error in tracing the tilt error might be such that occasional calibrations with a faint natural guide star might be necessary.

Another indirect advantage of the method results from the fact that the fringes extend over a wide field of view, and hence the cone effect is essentially taken care of by any MCAO technique. In fact the adoption of a set of fringes (laser or radio) is of interest in MCAO merely because the references will cover almost uniformly the whole patch of the sky that is going to be adaptively corrected. This uniformity results in a uniform correction of the turbulence, not only next to the reference beacons.

We have presented a novel technique that allows solving the global tilt problem in adaptive optics. This technique can be exploited not only in optical astronomy, but also in long base line optical interferometry (Gavel 1998), laser communications and laser power beaming to satellites. Another important application is in radio astronomy and in radio interferometry. In radio astronomy one needs to phase segmented mirrors and to sense the wave front (Serabyn et al. 1991). One of the most severe problems in radio interferometry is the lack of good phase calibration between the dishes (Thompson et al. 1986; Perley et al. 1985). The existence of reference hot spots is a significant step towards solving this problem. As in the visible regime, it should be set outside the central lobe of the radio dish or interferometer, but still within their field of view.

\section{References}

Baily, V. R. 1937, Nature, 139, 68, 838

Baharav, Y., Ribak, E. N., \& J., Shamir 1994, Opt. Lett., 19, 242

Baharav, Y., Ribak, E. N., \& J., Shamir 1996, J. Opt. Soc. Am. A, 13, 1083

Beckers, J. M. 1989, SPIE, 1114, 215

Belen'kii, M. S. 1995, SPIE, 2471, 289

Belen'kii, M. S., Karis, S. J., Brown, J. M., \& Fugate, R. Q. 1999, Opt. Lett., 24, 637

Bernhardt, P. A., Duncan, L. M., \& Tepley, C. A. 1988, Science, 242, 1022

Christou, J. C., Hege, E. K., Freeman, J. D., \& Ribak, E. 1986, J. Opt. Soc. Am. A, 3, 204

Esposito, S. 1998, SPIE, 3353, 468

Esposito, S., Ragazzoni, R., Riccardi, A., et al. 2000, Exp. Astr., 10, 139

Foy, R., Migus, A., Biraben, F., et al. 1995, A\&A, 111, 569

Foy, R., Tallon, M., Thiebaut, E., et al. 2000, J. Opt. Soc. Am. A, 17, 2236

Gaponov-Grekhov, A. V., \& Granatstein, V. L. (ed). 1994, Applications of high-power microwaves (Boston: Artech House)

Gavel, D. T. 1998, SPIE, 3350, 793

Gilmozzi, R., Delabre, B., Dierickx, P., et al. 1998, SPIE, 3352, 778

Glindenmann, A. 1997, PASP, 109, 682

Gurevich, A. V. 1972, Geomagnetizm I Aeronomiya 12, 631 (Geomagnetism \& Aeronomy 12, 556)

Gurevich, A. V. 1980, Sov. Phys. Uspekhi, 23, 862

Gurevich, A. V., Borisov, N. D., \& Milikh, G. M. 1997 (GBM97), Physics of microwave discharges (Reading: Gordon and Breach)

Happer, W., MacDonald, G. J., Max, C. E., \& Dyson, F. J. 1994, J. Opt. Soc. Am. A, 11, 263

Kagan, L. M., Kelly, M. C., Garcia, F., et al. 2000, Phys. Rev. Lett., 85,218

Kuo, S. P., \& Zhang, Y. S. 1990, Phys. Fluids B, 2, 667 
Lyakhov, S. B., Managadze, G. G., Petrov, M. S., \& Shlyuger, I. S. 1984, JETP Lett., 38, 658

Mountain, M. 1997, SPIE, 2871, 597

Parenti, R. R., \& Sasiela, R. J. 1994, J. Opt. Soc. Am. A, 11, 288

Perley, R. A., Schwab, F. R., \& Bridle, A. H. 1985, Synthetic Imaging, NRAO Workshop 13

Pilkington, J. D. H. 1987, Nature, 330, 116

Ragazzoni, R. 1996, APJ, 465, L73

Ragazzoni, R. 1997, A\&A, 319, L9

Ragazzoni, R., \& Rigaut, F. 1998, A\&A, 338, L100

Ragazzoni, R., Farinato, J., \& Marchetti, E. 2000, SPIE, 4007, 1076

Ragazzoni, R., Marchetti, E., \& Valente, G. 2000, Nature, 403, 54

Ragazzoni, R., Diolaiti, E., Farinato, J., et al. 2002, A\&A, 396, 731

Ribak, E. N. 1986, J. Opt. Soc. Am. A, 3, 2069-76

Ribak, E. N. 1997, Proc. ESO, 55, 186

Ribak, E. N. 1998, SPIE, 3353, 320

Ribak, E. N., \& Ragazzoni, R. 2001, Proc. ESO, 58, 281
Rigaut, F., \& Gendron, E. 1992, A\&A, 261, 677

Sandler, D. 1999, in Adaptive Optics in Astronomy, ed. F. Roddier (Cambridge University Press) 253

Serabyn, E., Phillips, T. G., \& Masson, C. R. 1991, Appl. Opt. 30, 1227

Sivaramakrishnan, A., Weyman, R. J., \& Beletic, J. W. 1995, AJ, 110, 430

Thompson, A. R., Moran, J. W., \& Swenson, G. W. 1986, Interferometry and synthesis in radio astronomy (New York: Wiley) Tyler, G. A. 1994, J. Opt. Soc. Am., 11, 358

Utlaut, W. F. 1975, IEEE, 63, 1022

Vikharev, A. L., Ivanov, O. A., Kim, A. V., \& Litvak, A. G. 1991, Proc. 20th Internat. Conf. Phenomena in ionized gases, 1, Pisa, Italy, 45

Wirth, A., \& Jankevics, A. 1992, Laser Guide Star Adaptive Optics Workshop, Albuquerque, ed. R. Q. Fugate, 606

Wuerker, R. F. 1997, Proc. ESO, 55, 16 